\title{
Registro e inventario de técnicas culinarias ancestrales de los días festivos en la provincia del cañar
}

\author{
Registration and inventory of ancestral culinary techniques of the holidays in the province of cañar \\ Registo e inventário de técnicas culinárias ancestrais de férias na província de Cañar
}

\author{
Ángel Guapisaca Vargas \\ investigacionyproduccion@sanisidro.edu.ec \\ https://orcid.org/0000-0002-8721-5885
}

María Herrera Calvo
competencias@sanisidro.edu.ec
https://orcid.org/0000-0002-5702-9555

\author{
John Valverde Minchala \\ titulacion@sanisidro.edu.ec \\ https://orcid.org/0000-0002-1149-7871
}

\author{
Ximena Alvear Racines \\ dirgastronomia@sanisidro.edu.ec \\ https://orcid.org/0000-0003-2973-537X
}

Instituto Superior Universitario San Isidro, Cuenca-Ecuador

Artículo recibido 5 de octubre 2021 / Arbitrado y aceptado 4 de noviembre 2021 / Publicado 30 de diciembre 2021

\section{RESUMEN}

El presente trabajo trata sobre "Registro e Inventario de técnicas ancestrales de los días festivos en la provincia del Cañar" (TCADF). Existen diversas técnicas en la preparación de alimentos en los días festivos que no se encuentran registradas de forma escrita y que pasa de generación en generación de manera verbal con la consiguiente pérdida de información. El objetivo de este trabajo se centra en realizar un inventario y posterior registro de las TCADF que todavía se pueden obtener de informantes previamente calificados de la zona de Cañar. La metodología de este estudio fue etnográfica y exploratoria se utilizó como herramienta y estrategia durante el proceso, para el levantamiento o recopilación de la información en campo. Además, como aporte metodológico los medios audiovisuales, forman parte activa en el registro testimonial. Se planifica períodos de convivencia con los diferentes informantes portadores de los conocimientos quienes proporcionaron la información de las prácticas culinarias, además de la observación directa que aportarán a la descripción y posterior registro de la manifestación del patrimonio inmaterial, objeto del trabajo que lo clasificamos en Instrumentos, técnicas de cocción y técnicas de conservación.

Palabras clave: Inventario; Registro; Técnicas culinarias ancestrales; Registro testimonial; Técnica audiovisual
ABSTRACT

The present study deals with "Registration and Inventory of Ancestral Techniques of Holidays in the Province of Cañar" (TCADF). There are various techniques in the preparation of food on holidays that are not recorded in writing and that are verbally passed down from generation to generation with the loss of information. The objective of this study is to carry out an inventory and a subsequent record of the TCADFs that can still be obtained from previously qualified informants in the Cañar area. The methodology of this study was ethnographic and exploratory. It was used as a tool and strategy during the process for the gathering or compilation of information in the field. In addition, audiovisual media are an active part in the testimonial record as a methodological contribution. Periods of coexistence are planned with the different informants possessing the knowledge which provides the information regarding the culinary practices, in addition to the direct observation that will contribute to the description and subsequent registration of the manifestation of the intangible heritage, the object of which we classify into instruments, cooking techniques, and preservation techniques.

Key words: Inventory; Record; Ancestral culinary techniques; Testimonial record; Audiovisual technique

\section{RESUMO}

O presente trabalho trata do "Registro e Inventário de técnicas ancestrais de férias na província de Cañar" (TCADF). Existem várias técnicas de preparação de alimentos nas férias que não são registadas por escrito e que são transmitidas de geração em geração verbalmente com a consequente perda de informação. O objetivo deste trabalho é fazer um inventário e posterior registro do TCADF que ainda pode ser obtido junto a informantes previamente qualificados na área de Cañar. A metodologia deste estudo foi etnográfica e exploratória, foi utilizada como ferramenta e estratégia durante o processo, para a recolha ou compilação de informação em campo. Além disso, como contribuição metodológica, os meios audiovisuais são parte ativa no registro testemunhal. São planejados períodos de convivência com os diferentes informantes portadores dos saberes que forneceram as informações sobre as práticas culinárias, além da observação direta de que contribuirão para a descrição e posterior registro da manifestação do patrimônio imaterial, objeto do trabalho que classificamos em Instrumentos, Técnicas de Cozinha e Técnicas de Conservação.

Palavras-chave: Inventário; Cadastro; Antigas técnicas culinárias; Registro de testemunho; Técnica audiovisual 


\section{INTRODUCCIÓN}

Las TCADF utilizadas en la gastronomía son el elemento básico de dichas festividades en las que se evidencian los procesos de preparación, que, por la solemnidad de la festividad, establece ya un rigor en su utilización y en el cuidado de elaborar la comida y bebida, propios del lugar de la celebración. Mediante investigaciones y búsquedas llevadas a cabo en bibliotecas de la zona, se percató que no existe un registro de estas técnicas, las que corren el riesgo de perderse en el olvido, pues las preferencias alimentarias van cambiando o modificándose por la globalización, la emigración entre otros factores. De esta manera, la tradición culinaria de estas técnicas, que ha sido el medio por el cual se ha heredado la preparación de estos platos, al no ser debidamente sistematizada puede hacer que estas técnicas de preparación desaparezcan.

Así mismo, es necesario indagar la influencia de creencias, costumbres y tradiciones en el consumo de productos nativos, no solamente como el hecho nutritivo o fisiológico de alimentarse, sino más bien cómo éstos han contribuido y han sido parte integral del desarrollo de los pueblos indígenas ancestrales y sus manifestaciones, entre ellas la gastronomía y sus respectivas técnicas.

Mediante la observación directa con grupos nativos de la provincia del Cañar de las TCADF, se obtuvo evidencias para los registros pertinentes. Estas técnicas van acompañadas de ciertas creencias que están relacionadas desde el cultivo hasta la preparación de alimentos, que se vinculan aparentemente con el rendimiento para el trabajo y longevidad, situación que da mayor significado y sentido a estas prácticas y que merece ser puesta en evidencia.

El propósito del estudio es identificar y rescatar las técnicas culinarias ancestrales de los días festivos (TCADF). Estás técnicas se utilizaban para la preparación de sus alimentos y productos de la dieta diaria de nuestros antepasados, los que al parecer proporcionabanuna buena nutricióny fortaleza para las intensas actividades agrícolas.

\section{Marco teórico}

El registro gastronómico e inventario de TCADF y su posterior socialización pretende llevar a cabo la recuperación de la memoria histórica, porque es parte del patrimonio intangible que, aparte de ser un elemento de identidad culinaria, es el origen del comportamiento fundamental en las economías sociales o comunitarias (1).

Según Encalada (2), el sincretismo entre las religiones precolombinas indígenas y el calendario litúrgico católico definen las festividades y éstos a su vez tienen una coincidencia con el calendario agrícola, sobre todo cuando se relaciona a las siembras y a las cosechas. Escobar (3) dice que toda fiesta se constituye a partir de un paquete de acciones y actuaciones realizadas por una colectividad en forma extraordinaria (no cotidiana), aunque generalmente periódica y más o menos codificada. Estas acciones recuerdan momentos fundamentales de la memoria 
común o propician situaciones esperadas por los participantes.

Quizás el concepto que mejor defina día festivo se refiera a actividades colectivas, extraordinarias generalmente periódicas y más o menos codificadas, carga simbólica vinculada a un espíritu de emotividad compartida. La fiesta, exalta la imagen de un nosotros y reafirma lazos de integración social (4). Por otra parte, se hace referencia al significado de gastronomía ancestral, para lo cual se ha revisado diferentes publicaciones, además de diálogos con diferentes actores de la población del sector de investigación; encontrando que la gastronomía ha sido fundamental en la vida no solo cotidiana de la población sino, es un punto de unión para lo social, laboral e incluso espiritual.

No se ha encontrado una definición concreta de lo que es la gastronomía ancestral, por lo que se podría considerar más bien que es aquella que procede de los antepasados, sin límite de generaciones, pero que perdura en la memoria colectiva, ocasionando que las comidas o platos se preparen con los ingredientes originales y utilizando las mismas técnicas de preparación. Éstas han pasado de generación en generación de forma oral, por lo que no se cuenta con un registro escrito sino más bien con la memoria colectiva de los habitantes del sector (1).

Al hablar de técnicas ancestrales, se hace referencia a las utilizadas antes y después de la conquista. Se conoce que, en la época prehispánica, las técnicas de preparación y preservación de alimentos eran básicamente la cocción directa al fuego colocando la comida directamente en las brasas o sobre piedras calientes y en ollas de barro. Para la preservación de los alimentos se utilizaba la técnica del asoleado, secado, ahumado, cocción y congelación (para deshidratar algunos tubérculos) (5). Las carnes por ejemplo (venado, llama, conejo, res) eran expuestas al sol untadas con sal, a fin de evitar su descomposición, en otros casos se utilizaba la técnica del ahumado ya que, principalmente los alimentos se cocían en leña y existía un trípode en el que se colocaba la carne o el pescado para ahumarla. El asoleado era otra forma de conservar los alimentos, principalmente de los granos que los secaban al sol tanto enteros como rallados como es el caso del maíz que se lo rallaba y secaba al sol, quedando así una especie de harina, misma que la utilizaban de diferentes formas, pero principalmente la hidrataban y fermentaban, dando como resultado una bebida que la ingerían en sus reuniones sociales y familiares (6).

Luego de la conquista española, se introducen nuevas técnicas de preparación ya que se introducen nuevos productos como el trigo, la cebada y carnes de animales que no eran nativos de esta zona. Por primera vez, se utiliza el molino para triturar los granos y producir harina en grandes cantidades, en casa se utilizan las piedras de moler. Las casas ya contaban con un lugar determinado para la cocina, por lo que el ahumado prevalece dentro de las técnicas de preservación y de cocción de los alimentos. Se introducen nuevas especies para condimentar las comidas, 
especialmente las carnes, incluido el cuy que era hasta entonces solo sazonado con sal. Aparecen también nuevas técnicas de cocción como son el asado en parrilla, frituras y cocina al vapor (7).

Posteriormente, se han sido fusionando técnicas, productos, condimentos y sabores, mismos que se han repetido de generación en generación. Lamentablemente al no existir un registro de esos saberes ancestrales, junto a otros factores como migración, globalización y el mismo hecho de crecimiento poblacional, ha inferido en la desaparición de muchas de estas técnicas culinarias ancestrales (8). Durante las visitas se constató que las personas que viven en el sector de investigación han cambiado sus costumbres, por ejemplo, ya no consumen chicha ya que es un proceso que toma varios días y para mayor comodidad se ingieren bebidas gaseosas. La disminución en el consumo de la chicha se aprecia desde la época de la conquista, pues se cambió el consumo de la chicha por el aguardiente ya que este permitía una forma de mantener sometido al pueblo indígena (9).

Antiguamente para endulzar se utilizaba el pulque (bebida obtenida del penco); en la actualidad se utiliza el azúcar o la panela ya que en el período hispánico se introdujo la caña de azúcar y por ende los productos fruto de su procesamiento (10).

Estos planteamientos ponen en evidencia una serie de razones que justifican la necesidad fundamental de preservar la memoria de las técnicas culinarias utilizadas por nuestros antepasados y que actualmente se están desvaneciendo.

\section{MATERIALES Y MÉTODOS}

El diseño de la investigación se definió como investigación no experimental cualitativa. Diseños transversales correlacionales/ causales: las causas y efectos ya ocurrieron en la realidad (estaban dados y manifestados). Se observó, se registró y se reportó.

Investigación de campo la investigación se centró en realizar el estudio donde el fenómeno se da de manera natural, de este modo se eligió la situación lo más real posible, los cuales fueron: Estudio de casos, donde se seleccionaron diferentes grupos para realizar la observación de los eventos gastronómicos culinarios. Estudio Etnográfico, donde se estudió la convivencia en la comunidad, con el objeto de observar, con una pauta previamente elaborada. Y cómo técnicas se recurrieron a la observación participante, la entrevista, el análisis documental y el autorreportaje.

La primera actividad consistió en identificar a los informantes clave de la Zona de Cañar que apoyen en el proceso de recopilación, revisión y construcción de las técnicas culinarias de los días festivos. Para esto de estableció los siguientes parámetros: Personas que realicen la preparación de los alimentos en la actualidad en los días festivos.

Las cocineras o Aucalanos, son mujeres que preparan los alimentos en la casa del prioste y varían entre 3 o 4, conjuntamente con el prioste reunirán los materiales e insumos que se utilizarán en la preparación de la comida para la fiesta. En algunos lugares el aucalano también aparece como un "encargado" de cuidar la casa y el servicio a los invitados a 
la casa. Es una persona a quien se le prohíbe consumir licor para que su función se realice a cabalidad. También tenemos la presencia de las Muñidoras: mujeres de entre 15 a 20 años que se desempeñan como ayudantes de la cocina y en los quehaceres de la casa del prioste como la limpieza, cuidado de los animales y demás actividades mientras dure la fiesta, son los aliados estratégicos de la aucalanos.

Se contactó con ellos mediante el Ministerio de Turismo y Asociaciones cuya actividad se relacionaba con la producción de alimentos y se concretó en una base de datos. Se identificó un número significativo de informantes a los que denominaremos "informantes calificados". El registro de los informantes se documentó con la identificación en la que consta: nombre, edad, sexo, estado civil, información de contacto, comunidad, dirección, teléfono, correo electrónico.

Se definió también los días festivos en los que la preparación de los alimentos aporta el valor simbólico a la celebración que se caracteriza porla ritualidady que esta ritualidad está fundamentada en sus creencias propias. A estas creencias hay que incluir el papel de la Iglesia que jugó en el adoctrinamiento de la población, confrontando las celebraciones propias que se desarrollaba según el calendario agrícola con el santoral católico produciéndose el sincretismo religioso.

Los días festivos están asociados a las grandes fechas del calendario agrícola es decir siembras, maduración y cosecha de los cultivos, de los cuales definimos el Inti Raymi,
Killa Raymi, Capac Raymi, Ayamarca Raymi, Taita Carnaval, siendo la más importante el Inti Raymi (11). Se definió este grupo de festividades porque en ellas se encuentra un factor común en los alimentos y su preparación. No se consideró otras festividades, como el Corpus Cristi, las fiestas de cantonización, fiestas de santos como San Juan o de la Virgen de la Nube porque el contenido gastronómico ya no se ejecutaba con los criterios ancestrales o simplemente su celebración había sufrido un debilitamiento, y ya casi no se celebran (12).

Con este criterio se observó en las diferentes festividades, tanto la propuesta gastronómica como la técnica consisten fundamentalmente en el consumo del siguiente menú: Papas, cuy, gallina, carne de cerdo, res, maíz, granos tiernos y maduros, ají, chicha de jora. Así mismo dependiendo de la época del año se obtuvo información de las técnicas para el procesamiento de dichos alimentos.

La metodología y algunos resultados estuvieron condicionados por la Cosmovisión Andina y su importancia para entender los comportamientos en los procesos culinarios.

Los instrumentos de recolección de datos fueron: Video, audio, fotografía, formatos, apuntes, cuaderno o diario de campo.

\section{RESULTADOS Y DISCUSIÓN}

Durante las festividades observadas en diferentes sectores y en diferentes épocas se sintetizaron las manifestaciones que son comunes en estos eventos en cuanto a las técnicas culinarias de cocción y también se realizó el registro de los instrumentos y 
algunas técnicas de conservación empleados en los eventos.

Los resultados obtenidos los clasificamos de la siguiente manera:

- Instrumentos: Fogón, ollas de barro, tiestos, (tostador de metal).

- Técnicas de cocción: Tostar, hervir, asar, moler.

- Técnica de conservación: fermentado, deshidratado, secado, molido.

Alimentos comunes en las fiestas: Tasno, caldo de gallina, máchica, chicha de jora, cuy, papas.

\section{El fogón}

El fogón se instala en la parte central de la cocina, a diferencia de los fogones de uso diario que ya disponen de un espacio definido y su tamaño es afín a la cantidad de preparaciones a realizar de acuerdo al número de miembros de la familia, el fogón debido a la cantidad de alimento a cocinar, funciona mejor realizarlo en el piso debido al tamaño de los contenedores utilizados para la cocción. Se limpia bien el espacio en donde se instalará el fogón, luego lo humedecen de manera que no se levante polvo al momento "soplar" sobre las "tarallas" (cortezas de árboles, hojas de pencos, y ramas secas que se han desprendido de sus plantas) y leños encendidos hasta obtener el fuego. Para los cañaris el espacio terrestre es el equilibrio entre el "arriba" y el "abajo", también entre el hombre y lo numinoso, entre el frío de la noche y lo cálido del día. la responsabilidad del ser humano es mantener y no quebrantar ese equilibrio, por ello es necesario cuidar su conducta y llevar a cabo elaborados rituales y ceremonias como "pedir permiso a la tierra", este acto de humedecer el piso y recitar una oración imperceptible cumple también esa función, no hacerlo significa una agresión a la madre tierra.

La misma actitud de respeto y de pedir permiso a la naturaleza se mantiene el momento para ingresar en ella o tomar algo de la misma. Así sucede con el abastecimiento de leña chaguarqueros, tarallas, hojas de penco secos, etc. Estos elementos que se encuentran fuera del ciclo vital y que lo pueden recoger sin tener que "agredir" a la naturaleza, se diferencia cuando tienen que tomar leña, que, de igual manera, por respeto a la madre tierra solicitan el permiso respectivo. "La leyenda del Chavar" y "La Historia del Carbonero" sustentan esta creencia.

Para encender el fogón es importante el rito que deben cumplir aquellos que en primera instancia van a internarse en los bosques a traer la leña y luego quienes organizan el fogón en la cocina. Es importante cumplir con este paso, porque en lo más profundo del pensamiento y la cosmovisión andina, todo es un ser viviente, todo tiene energía y cuando se cumple con el ritual, con el rezo, una vez cumplido, este proceso de instalación del fogón se procede con la calma y la tranquilidad.

Según los informantes hay varias formas de solicitar "permiso a la madre tierra", es decir hay varias formas de comunicación de ella con los seres vivos y antes de tomar sus productos se cumple un ritual que siempre empieza con 
expresar lo que se siente por ella. Al parecer el tipo de rezo es mpas de inspiración individual que tener una oración que lo digan todos.

\section{Las ollas de barro y los tiestos}

Son hechos de arcilla quemadas en hornos de leña a una temperatura aproximada entre 500 a 600 grados centígrados, algunas son "bruñidas" o pulidas con piedras de río con la finalidad de impermiabilizar. También son curadas o les hacen "arishcas" (especie de sellado de las porosidades propias de la arcilla cocida, se lo hace con la finalidad de minimizar la porosidad de manera que no guarde memoria de los sabores de los alimentos que se cocen en ellas) sometiéndoles al fuego y luego colocan agua hirviendo. Hay casos en los que frotan ajo y luego agua hirviendo, y otros igualmente friegan con cáscaras de guineo. Según la creencia es para emporar y para que la cocción de los alimentos sea más rápida.

Los platos y jarros que también son de barro, no son sometidos a este proceso de quema y en algunos sectores los utilizan con un vidriado que es hecho a base de plomo.

No se encontró ollas ni tiestos producidos con tierras y tecnologías del mismo lugar, objetos elaborados con la técnica ancestral llamada "golpeadores o huactana", sino más bien los recipientes actuales eran ya comprados en Jatumpamba, San Miguel de Porotos, un lugar conocido tradicionalmente por la elaboración de objetos de barro.

\section{Tostador de metal}

Contenedor hecho de latón, sin embargo, en tiempos pasados se lo realizaba en tiestos de barro, traídos desde San Miguel de Porotos. No se pudo registrar el tiesto ya que en ninguno de los lugares visitados lo utilizaron. El contenedor tiene un tamaño de $60 \mathrm{~cm}$ de ancho por 120 de largo, y cuya altura es de $10 \mathrm{~cm}$, no tiene juntas de soldadura, cuenta solamente con dobleces y unido con remaches de metal. Por los extremos tiene dos asas para sujetar el contenedor y poder mover sobre el fuego.

\section{Idupo}

Para mover los granos que se están tostando se utiliza un accesorio que lo llaman "idupo" para mantener los granos en movimiento, y de esta manera evitar que se quemen. Es importante que los granos estén literalmente secos. El Idupo es una vara de carrizo o madera en cuyo extremo se ha cubierto con un pedazo de cuero de borrego, amarrado con cabuya obtenido de las fibras secas de penco.

Cucharas de madera. De diferentes tamaños y funciones, se los utiliza para mover los alimentos durante la cocción.

\section{Técnicas: Tostar, hervir, asar, moler}

\section{Tostar}

Esta técnica consiste en cocer el alimento sobre el tostador, sin ningún aditamento graso. Una vez instalado el fogón se coloca 
el tostador y sobre él se coloca el grano seco. Si es para el tostado, es solamente el maíz blanco o "chulpi" y se lo somete al proceso de tostado durante un tiempo aproximado de una hora, aunque esto depende de la cantidad del maíz a tostar. En el caso observado se tostó medio quintal de maíz blanco. Mediante este proceso se extrae la humedad del alimento y su coloración va cambiando hasta llegar a un color dorado o marrón y con una consistencia crujiente.

Mientras el maíz se va "tostando" su coloración también. Es importante no dejar de mover para lograr una uniformidad en la cocción. En algunos lugares colocan grasa de cerdo para mejorar el sabor del tostado. En el caso del tasno en el que se utiliza habas secas, maíz seco, como principales ingredientes. El proceso es el mismo, solamente que el "tostado" no concluye con la cocción total de los granos, sino que se llega a un porcentaje de cocción del $50 \%$ y se tuestan cada grano por separado.

Se interrumpe el proceso de tostado en momento en que visualmente se considera que el grano está listo para el siguiente proceso, que es juntar con papas cocidas o mellocos, conformando finalmente con ellos el Tasno, llamado por los mismo como "comida para la resistencia". La propuesta gastronómica con esta técnica la encontramos en el tostado y en el tasno. El tostado tiene un porcentaje de cocción total de manera que el maíz maduro o seco llegue a un color anaranjado oscuro.

\section{Hervir}

Se constató con claridad dos tipos de cocción mediante el hervido. La primera que arte desde al agua fría, es decir que los alimentos se colocan desde el inicio en la olla de barro, junto con el agua, y se someten al fuego hasta llegar al punto de ebullición y ahí permanece el tiempo que necesite para la cocción. La mayoría de tubérculos como la papa, la oca, la mashua, los mellocos. También productos como el maíz (mote) o cebada.

La otra forma de cocer consiste en calentar el agua hasta llegar al punto de ebullición, momento en el que colocan los alimentos, de esta manera pueden tener un mejor control sobre la cocción. Este proceso se observó en momento de cocer carnes, ya sea de res, cerdo, pollos, borrego, cuy. Los recipientes utilizados como contenedores para la cocción son ollas de barro.

En los días festivos la sopa principal que se hace es el caldo de gallina, presente en la mayoría de fiestas. También y con menor presencia hay caldos de res, borrego, cuy, y sopas.

El Caldo de gallina tiene un proceso simbólico desde el faenamiento del animal. Se escoge las aves dependiendo a quienes se ofrezca la comida en el día festivo. Por ejemplo, si es para el público que asiste a la festividad o son invitados sin ningún vínculo íntimo simbólico, da igual el tipo de ave a sacrificar. Sin embargo, en el momento del sacrificio, al igual que en otras ocasiones, se 
pide permiso a la madre tierra (naturaleza) por tomar esa vida. Mientras se ejecuta al ave y se quitan las plumas, éstas son reunidas por "puñados" y esparcidos por el campo a manera de restitución y agradecimiento con el inframundo, definido en sus creencias. Cada puñado igualmente está acompañado de una oración individual.

Cuando los invitados se reúnen en la casa del prioste, y son del círculo del agrado de los dueños de casa, los animales, en este caso la gallina tiene un antecedente simbólico importante, tanto para el que sacrifica como para el que se va a servir. El ave escogida debe reunir los colores del inframundo o del supramundo, representados en colores blanco o negros, y también se incluyen los pollos que tengas esos dos colores. La unión de estos dos mundos concretados en este espacio real de la existencia humana y ofertados en el animal sacrificado es una muestra de afecto y cariño profundo para los que participan de esta comida. El desarrollo de la preparación (receta) para el caldo es común en todos los lugares.

\section{Asar}

Exponer el alimento al calor que emana de la combustión de carbón vegetal o brazas de leña. Esta técnica tiene diferentes maneras de usarse. En la que más se utiliza en la preparación del cuy, uno de los ingredientes principales en las todas las festividades de la provincia del Cañar, usado desde tiempos del incaico hasta la actualidad. Al igual que la preparación de la gallina, el cuy también tiene el mismo simbolismo en cuanto al color. Y los cuyes escogidos para el círculo íntimo tienen estas características cromáticas. Cuyes blancos y negros y cuyes marrones, en ellos se sintetizan las tres realidades de la cosmovisión andina, el Uku pcha, el Hanna Pacha y el Urcu pacha. Tener una celebración con esta propuesta de los cuyes, y ser invitado a este evento, dice mucho del aprecio de quienes convidan hacia los que son invitados.

Una vez escogidos los cuyes para el faenamiento se procede con el ritual. Al cuy se lo coge entre los dedos de la mano por el cuello, y se lo sacude fuerte una sola vez, de manera que el cuy queda aturdido entonces se procede a cortarle la yugular y hacer que se desangre. Se lo toma por las patas y se lo coloca de cabeza para que la sangre salga. Luego de muerto el cuy se le pela con agua caliente, mientras quita la lana, esta se va reuniendo en la mano hasta formar unas esferas de lana, que así mismo al compás de una oración se esparce las lanas por el rincón de la casa en señal de pedir fecundidad y bendiciones en la producción de cuyes.

El cuy una vez faenado es adobado con una mezcla de ajo, sal y comino, básicamente. En algunos lugares hacen este proceso hasta con dos días de preparación para que los sabores del aliño penetren el cuy.

Para asar el cuy se lo empala en un palo de madera, que algunas veces tiene hasta dos espacios para dos cuyes y luego es suspendido y sometido a la exposición del calor emanado por el carbón ardiendo a una distancia considerable de manera que se garantice 
la cocción sin quemarse. Se puede también sumergir ciertos alimentos en la brasa o colocarlos directamente sobre ella.

\section{Moler}

Ají molido en piedra. La técnica utilizada en el proceso es muy parecida en todos los lugares, las piedras de moler son similares y constan de 2 partes: una superior y otra inferior. La inferior generalmente tiene una forma redondeada y con una concavidad central semi profunda, ésta por lo general va asentada en el suelo, aunque hay personas que las apoyan sobre un banco o una mesa ya que también depende del tamaño de la piedra; otra variación es que la piedra inferior es completamente plana. La procedencia de las piedras según testimonio es que las sacaron del río hace muchos años, muchas de ellas son heredades de sus madres y/o abuelas. La piedra superior tiene generalmente forma de media luna y que es más o menos del tamaño de la concavidad de la piedra inferior; unas con la parte superior lisa y la inferior convexa que es la que va a moler el ají, otras con la parte superior cóncava y la inferior convexa, se la maneja con ambas manos tomándola por los extremos y realizando movimientos de vaivén de derecha a izquierda, estos son rítmicos y enérgicos ya que realizan la presión respectiva para poder moler los ingredientes hasta formar una pasta.

En el caso de las piedras pequeñas, se utiliza una piedra superior con forma redonda u ovalada, se la maneja con una sola mano y en este caso los movimientos son circulares y de adelante hacia atrás en relación con la posición del cuerpo de la persona que muele.

En una casa pueden existir varias piedras, dependiendo del uso que se les quiera dar, por ejemplo: hay piedras para moler granos secos y tostados que generalmente se diferencias por la parte plana de la piedra base, otra para moler ajo y condimentos y una exclusivamente para el ají.

La máchica molida en piedra se lo realiza en la casa de personas de escasos recursos, y en comunidades que distan de otros donde tienen molinos modernos. El proceso consiste primero en el aventado de la cebada, que no se considera una técnica culinaria propiamente, aunque se observa el mismo procedimiento en el maíz y en el trigo, cuyo propósito es separar las impurezas como cascarillas que han quedado del trillado. La "técnica" consiste en tomar un poco de del producto, ya sea cebada, trigo, maíz, u otros granos, con las manos y levantar a cierta altura para dejar caer sobre un recipiente-contenedor, durante la precipitación de los granos, la persona que avienta, sopla de manera que las partículas más livianas se apartan de las más pesadas que finalmente van a dar en el contenedor.

Una vez la cebada limpia de las impurezas, se procede al tostado de los granos en el tostador con el uso del idopu (instrumento para mover los granos en el tostador, mientras se tuestan) para remover. Hay un punto en donde se suspende el tostado. El criterio para considerar que el grano está listo para ser molido es cuando éste adquiere un color marrón. El sabor de la máchica está definido 
por el grado de tostado de los granos.

Los granos tostados son llevados a una piedra plana en cuyos extremos se sitúan, la persona que va a moler y en el otro extremo un recipiente de madera (batea) que receptará la harina o polvo de cebada molida, conocida con el nombre de máchica. La persona que realiza el molido presiona los granos tostados con una piedra igualmente plana, sobre la otra superficie de la piedra que generalmente se encuentra en el suelo. Con movimientos de atrás hacia adelante se produce el molido de los granos, en cada movimiento se toma una cantidad de granos que abarque el tamaño de la piedra que se mueve y se va acumulando en el otro extremo. Este proceso se vuelve a repetir hasta que la granulometría del molido sea considerada la adecuada. Cuando esto sucede, se deja de acumular el molido en él un extremo y se deja caer el polvo definitivo en la batea. Entonces la máchica está lista para finalmente pasar a cernir.

El cernido se lo realiza en un cedazo, la mayoría son de chapa de madera como contorno y la malla es construida de crin de caballo. Solamente en una comunidad se encontró un arnero (colador), que es de cuero de borrego con perforaciones realizadas con objetos punzantes generalmente hechos de madera.

La machica cernida es preparada con grasa de chancho, sal y perejil, llamada "sarasango" y consumida cuando se faena el chancho. A manera de postre, la máchica se mezcla con rayadura de panela traída desde San Antonio de Paguancay consumiéndose como postre en algunas localidades durante las fiestas.
Hay otros usos que las personas del Cañar le han dado a la máchica. Por ejemplo, han creado galletas con formas de animales o muñecos que sirven a parte de jugar con ellos, como colación para que los niños lleven a sus tareas en el campo o a sus actividades escolares. Se encontró a la machica también usada en sopas, coladas, pan, etc., pero no se manifiestan en los días festivos.

\section{Fermentar}

La Chicha de Jora es el producto que aparece en todas las fiestas, especialmente en el Inti Raymi (13), y es elaborada por los integrantes de las comunidades andinas, no solamente del Cañar. Su ingrediente es la jora o maíz germinado es utilizado como bebida ceremonial.

Si bien su origen se lo atribuye a la leyenda de una fermentación casual del maíz almacenado. La leyenda más agradable alrededor de esta bebida típica se le atribuye el descubrimiento casual de la chicha de jora cuando por las lluvias se dañó el maíz almacenado en las bodegas en las que se guardaban los granos y se fermentaban.

Como tradición familiar en las comunidades indígenas de la Sierra se consume como líquido refrescante en las mingas, para dar la bienvenida a una persona importante, e incluso brindarla en conmemoraciones como matrimonios, bautizos, Navidad, Carnaval y otros.

El procedimiento comienza con la germinación del maíz: al maíz hay que poner cinco días en el agua para el remojo, luego se lo coloca en una cama hecha de altamizo y 
chilca, también en algunos lugares lo colocan sobre hojas de penco, se dice que según la cama adquiere diferentes sabores. La función principal del penco es para mantener la humedad del "microclima" que facilitaría la germinación del maíz (brote) se vuelve a tapar con las hojas partidas del penco y sobre este la chilca y como un guardián de los insectos la altamisa como última capa.

Luego de germinado se procede al secado, que se lo hace directamente por la exposición al sol, se lo tiende en los corredores de las casas o patios hasta que el maíz pierda humedad. El control del secado es muy importante, ya que la presencia de la no eliminación adecuada de la humedad puede afectar la calidad del maíz, por tanto, la calidad de la chicha.

Una vez secos los granos de maíz de manera uniforme se procede a la molienda, para obtener la harina que es el ingrediente base para la chicha y es el que será sometido a la cocción. Durante la cocción se elimina cualquier tipo de gérmenes patógenos o agentes tóxicos presentes, también se obtienen características en el sabor y en el color de los alimentos. En esta etapa también se condimente y saborizan con ingredientes como clavo de olor, canela, pimienta panela.

Una vez que ha ocurrido el proceso fermentativo, el producto terminado, al que se le somete a un paso final que es el filtrado, o cernido en cedazos construidos con crin de caballo (pela largo y grueso que crece en el cuello de los caballos) cuyo objetivo es para retener partículas indeseables y restos de levaduras de manera que la presencia de la chicha es mejor.

\section{Discusión}

Se pone a consideración la definición de ancestral con la que se presenta este estudio. Lejos de situarle cronológicamente, ha sido mucho más funcional declarar que dicho conocimiento se obtuvo de personas que mantienen vivo el recuerdo y que de alguna manera estén en la posibilidad real de ejecutar las técnicas, independientemente de la edad, aunque se constó que la mayoría de informantes son mayores de edad y que son quienes dirigen las faenas de la preparación de la comida en los días festivos. Personas que presenciaron y testificaron los preparativos desde su niñez y que en ellos se depositó dicha enseñanza y que han venido ejecutando hasta la actualidad.

Otro concepto a definir es el día festivo en la provincia del Cañar. Recordemos que las actuales festividades son productos de la mezcla de creencias propias del pueblo cañari y que se encuentra mezclado con la religión produciéndose el sincretismo que, si en ningún caso es la anulación de las manifestaciones, sino que emergen como un complemento. Sin embargo, la filosofía de la cosmovisión andina es quien en última instancia define el sentido ceremonia y ritual de los días festivos. Existe mucho ocultamiento en las creencias antiguas. No se manifiesta en el verdadero sentido en que sus antepasados vivieron, sin más bien su transformación obedece al acople que sienten que deben tener frente a la modernidad y en ese transcurso se va debilitando algunos detalles como las leyendas y plegarias que en algún momento fueron vitales para la preparación de los alimentos. 
La propuesta de inventario y registro de las técnicas culinarias ancestrales, y su delimitación conceptual quedan planteados como un trabajo a definir. Existen utensilios que pertenecen a la modernidad y otros que en algún momento pertenecieron también a su "modernidad" y por la fuerza de la costumbre en el uso o la desaparición de los mismos, han llegado a integrarse en el dinamismo de las actividades gastronómicas. Por ejemplo. el tostador de barro que hoy lo realizan en una tostadora de metal, con remaches, o la misma cebada que con el paso del tiempo ha sido un alimento que se constituyó importante en la dieta cañari, comenzó como un alimento extranjero y que en el tiempo ha ido asumiendo su ancestralidad hasta llegar a nombrarle, actualmente, como tal.

Los alimentos preparados y presentados como menús, a pesar de lo expuesto en cuanto a técnicas, no ha perdido el simbolismo de la ritualidad y la celebración. El entender esta dinámica hará posible cumplir con la intención de salvaguardar la memoria histórica del pueblo cañari y apoyar en el fortalecimiento de su identidad.

\section{CONCLUSIÓN}

El recorrido por diferentes lugares de la provincia en busca de información de manera que testifique los procesos que se llevan a cabo en la actualidad, nos deja como resultado los siguientes criterios:

Las técnicas no se manejan en la actualidad con los utensilios e instrumentos que mucho de los informantes recuerdas, pero que hoy en día no se los consigue, ya que hay procesos más rápidos y seguros. El desarrollo gastronómico culinario de la fiesta si bien mantiene el simbolismo original, tiene el peligro de quedar encerrado en un concepto como folclor, como algo para mostrar a los turistas. Incluso alguno de los informantes ha visto la ocasión de vender la información y esta actitud influye en la consecución de evidencias. El trabajo queda planteado, se experimentó una dinámica en los acontecimientos, es decir los resultados se declararán en un determinado tiempo. Quizá más adelante aparezcan nuevas formas de llevar a cabo el proceso culinario de las fiestas.

\section{REFERENCIAS BIBLIOGRÁFICAS}

1. Unigarro CPatrimonio Cultural Alimentario. Quito, Ecuador: 2010

2. Encalada O. La Fiesta Popular en el Ecuador. Cuenca, Ecuador: CIDAP.2005

3. Escobar Ticio. 2003 Fiestas populares tradicionales e integración latinoamericana. Disponible en: https://biblio.flacsoandes.edu. ec/libros/digital/40032.pdf

4. Pereira J. La Fiesta Popular Tradicional del Ecuador. Quito, Ecuador: Fondo Editorial del Ministerio de Cultura. 2009

5. Gutiérrez R. Arte Latinoamericano del siglo XX: Otras historias de la historia. Prensas Universitarias de Zaragoza. 2005

6. Testimonio de Garzón, M. Antropólogo de la provincia del Cañar

7. Garzón M. comunicación personal 20 septiembre 2018

8. INEC. Resultados provinciales: Cañar. 2010. Disponible en: http://www.ecuadorencifras. gob.ec

9.Testimonio de Garzón M. Antropólogo de la provincia del Cañar 
10.Testimonio de Pichazaca, M. Habitante del sector Picotera

11. Ruiz E. Soliz, D. El Turismo Comunitario en Ecuador. Desarrollo y Sostenibilidad Social. Quito, Ecuador: Producciones Digitales AbyaYala. 2007
12. Ministerio de Cultura y Patrimonio. Inti Raymi, Fiesta del Sol y la Cosecha, en Ingapirca. 2007. Disponible en: http://www. culturaypatrimonio.gob.ec

13. Viajandox. Fiesta del Kapac Raymi. 2018. Disponible en: https://www.ec.viajandox.com 